\title{
Solution of Wave Equations on Transmission Lines where Leakage to Ground on the Line is Negligible
}

\author{
Michael Olufemi OKE \\ Department of Mathematical Sciences, Ekiti State University, Ado, Ekiti, Nigeria
}

Email address:

femioke91@gmail.com

To cite this article:

Michael Olufemi OKE. Solution of Wave Equations on Transmission Lines where Leakage to Ground on the Line is Negligible. American Journal of Applied Mathematics. Vol. 3, No. 3, 2015, pp. 124-128. doi: 10.11648/j.ajam.20150303.18

\begin{abstract}
This paper presents the solution of wave equations on transmission lines where leakage to ground on the line is very small. As a result of the leakages to ground on the transmission lines which are negligible, the conductance and the inductance, which are responsible for leakages on the line, are set to zero in the model of the general wave equation of the transmission line. The Laplace transform method was now applied to transform the resulting partial differential equation into ordinary differential equation and the method of variation of parameters was used to get the particular solution to the problem.
\end{abstract}

Keywords: Leakage to Ground, Initial Value Problem, Wave Equations, Transmission Lines

\section{Introduction}

Transmission lines are basically electrical circuits having distributed constants (or parameters. These parameters include the line resistance, line inductance, line capacitance and shunt conductance. The performance of a transmission line depends upon these parameters to a considerable extent. Every electrical conductor offers opposition to the flow of current and this opposition is called the resistance of the conductor. The resistance is distributed uniformly along the whole length of the line. It is the most important cause of power loss on the transmission line. The capacitance causes a charging current to flow in the transmission line. Any two conductors separated by an insulating medium constitute a capacitor or a condenser [1] and [2]. Series inductance mainly governs the power transmission capacity of the line. When an alternating current flows through a conductor, a charging flux is set up which links the conductor [1]. The conductors therefore posses inductance due to these flux leakages. The inductance is also uniformly distributed along the whole length of the transmission line. Inductance offers opposition to the flow of varying current in a circuit [1]. This is different from resistance which offers opposition to the flow of both steady (direct) and varying (alternating) current. The opposition to the flow of varying current, as a result of inductance, is called voltage drop. The shunt conductance is mostly due to leakages over the insulator [1]. Just like other transmission parameters, it is also uniformly distributed over the total length of the transmission line. The line inductance and shunt conductance are the parameters responsible for leakages along the transmission lines. In this paper, the line inductance and shunt conductance are therefore set to zero in the general wave equation of transmission lines to get the mathematical model which was solved by using the Laplace transform method.

The Laplace transform method has a lot of applications in initial and boundary value problems of differential equations arising in physics, mathematics and engineering. It originated from the work of Oliver Heaviside who found it useful to solve the equation of electromagnetic theory [3]. Integral transform methods which include the Laplace transform, Fourier transform, Hankel transform, to mention a few, has been applied to solve a lot of problems in differential equations in the field of science and engineering. [4] applied the Fourier and Hankel transforms to solve some boundary value partial differential equations. [5] used the Hankel transform method to solve some boundary value problems in their paper. The choice of a particular transform to be used for the solution of a differential equation depends upon the nature of its initial or boundary conditions [6]. In this paper, the Laplace transform method was used to transform the derived partial differential equation, in the mathematical model for the wave equation on transmission lines where leakage to ground on the line is negligible, to ordinary differential equation. The method of variation of parameters was now used to get the particular solution for the problem. 


\section{Materials and Methods}

Let us considered the equivalence circuit of a transmission line having length $\Delta x$ with resistance $R \Delta x$, capacitance $C \Delta x$, inductance $L \Delta x$ and conductance $G \Delta x$ as shown in figure 1 below.

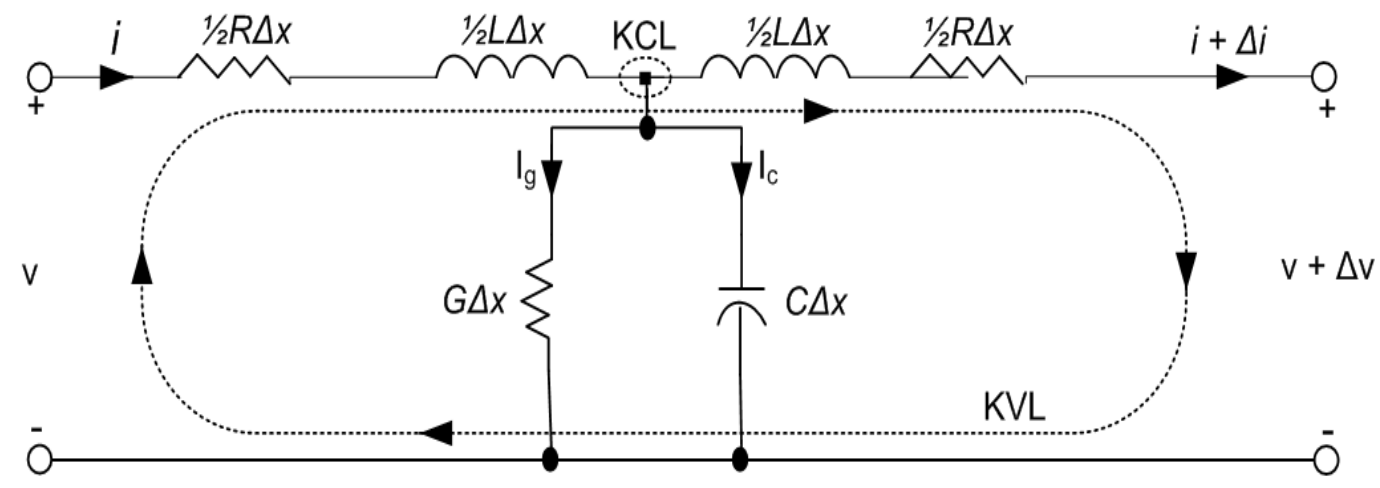

Figure 1. Equivalent Circuit of a Transmission Line.

Applying the Kirchhoff's voltage and current laws to the symmetrical network of figure 1 and simplifying accordingly, we have

$$
\frac{\partial v}{\partial x}=-\left[R i+L \frac{\partial i}{\partial t}\right]
$$

and

$$
\frac{\partial i}{\partial x}=-\left[G v+C \frac{\partial v}{\partial t}\right]
$$

Equations (1) and (2) above describe the evolution of current and voltage on a lossy transmission line [7] and [8].

Differentiating (1) with respect to $\mathrm{x}$ and (2) with respect to $t$ and simplifying the result, we have

$$
\frac{\partial^{2} v}{\partial x^{2}}=L C \frac{\partial^{2} v}{\partial t^{2}}+L G \frac{\partial v}{\partial t}+R\left[G v+C \frac{\partial v}{\partial t}\right]
$$

Differentiating (1) with respect to $t$ and (2) with respect to $\mathrm{x}$ and simplifying the result, we have

$$
\frac{\partial^{2} i}{\partial x^{2}}=L C \frac{\partial^{2} i}{\partial t^{2}}+C R \frac{\partial i}{\partial t}+G\left[R i+L \frac{\partial i}{\partial t}\right]
$$

Equations (3) and (4) are hyperbolic partial differential equations which represent the general wave equations for a lossy transmission line [7] and [8].

When leakages to ground on a transmission line are negligible, the conductance and the inductance on the line are set to zero because they are the factors responsible for leakages on the line [1], [2] and [9]. Therefore, setting G and $\mathrm{L}$ to zero in equation (3), we have

$$
\frac{\partial^{2} v(x, t)}{\partial x^{2}}=R C \frac{\partial v(x, t)}{\partial t}
$$

Similarly setting G and L to zero in equation (4), we have

$$
\frac{\partial^{2} i(x, t)}{\partial x^{2}}=C R \frac{\partial i(x, t)}{\partial t}
$$

Let $\alpha=C R$, so that equation (6) now becomes

$$
\frac{\partial^{2} i(x, t)}{\partial x^{2}}=\alpha \frac{\partial i(x, t)}{\partial t}
$$

Equation (7) can now be solved together with the following initial conditions

$$
i(x, 0)=f(x), i_{t}(x, 0)=g(x)
$$

where $i$ is the current through the conductor, $f(x)$ is the initial value of the current, $g(x)$ is the initial speed of the current and $0<x<k$.

Taking the Laplace transform of (7) with respect to t, we have

$$
s^{2} I(x, s)-s i(x, 0)-i_{t}(x, 0)=\alpha \frac{d^{2} I(x, s)}{d x^{2}}
$$

Putting the initial conditions, we have

$$
\alpha \frac{d^{2} I(x, s)}{d x^{2}}-s^{2} I(x, s)=-[g(x)+s f(x)]
$$

This now gives

$$
\frac{d^{2} I}{d x^{2}}-b^{2} I=\operatorname{cy}(x)
$$

where $b^{2}=\frac{s^{2}}{\alpha}$ and $c y(x)=\frac{-[g(x)+s f(x)]}{\alpha}$

The general solution of (11) is given by

$$
I=I_{c}+I_{p}
$$

where $I_{c}$ is the complementary function and $I_{p}$ is the particular integral.

Solving the associated homogeneous differential equation for (11), we have the complementary function as

$$
I_{c}(x, s)=k_{1} I_{c_{1}}(x, s)+k_{2} I_{c_{2}}(x, s)=k_{1} e^{b x}+k_{2} e^{-b x}
$$

Using the method of variation of parameters, we seek a particular solution of the form

$$
I_{p}(x, s)=U_{1}(x) I_{c_{1}}(x, s)+U_{2}(x) I_{c_{2}}(x, s)
$$

where $I_{c_{1}}(x, s)=e^{b x}, I_{c_{2}}(x, s)=e^{-b x}, U_{1}(x)=\int \frac{w_{1}}{w} d x$ and $U_{2}(x)=\int \frac{w_{2}}{w} d x,[10]-[13]$.

Values of $w, w_{1}$ and $w_{2}$ are obtained from the determinants below 


$$
\begin{aligned}
& w=\left|\begin{array}{cc}
e^{b x} & e^{-b x} \\
b e^{b x} & -b e^{-b x}
\end{array}\right| \\
& w_{1}=\left|\begin{array}{cc}
0 & e^{-b x} \\
c y(x) & -b e^{-b x}
\end{array}\right| \\
& w_{2}=\left|\begin{array}{cc}
e^{b x} & 0 \\
b e^{b x} & c y(x)
\end{array}\right|
\end{aligned}
$$

We can easily see from (15), (16) and (17) that $w=-2 b$, $w_{1}=-c y(x) e^{-b x}$ and $w_{2}=c y(x) e^{b x}$.
Therefore $\quad U_{1}(x)=\int \frac{c y(x) e^{-b x}}{2 b} d x \quad$ and $\quad U_{2}(x)=$ $-\int \frac{c y(x) e^{b x}}{2 b} d x$.

Substituting the values of $U_{1}(x), U_{2}(x), I_{c_{1}}(x, s)$ and $I_{c_{2}}(x, s)$ in (14), we have the particular solution as

$$
I_{p}(x, s)=\frac{e^{b x}}{2 b} \int c y(x) e^{-b x} d x-\frac{e^{-b x}}{2 b} \int c y(x) e^{b x} d x
$$

The general solution to (11) is therefore

$$
I(x, s)=k_{1} e^{b x}+k_{2} e^{-b x}+\frac{e^{b x}}{2 b} \int c y(x) e^{-b x} d x-\frac{e^{-b x}}{2 b} \int c y(x) e^{b x} d x
$$

Substituting the values of $b$ and $\operatorname{cy}(x)$ in (19), we have

$$
I(x, s)=k_{1} e^{\frac{x s}{\sqrt{\alpha}}}+k_{2} e^{\frac{-x s}{\sqrt{\alpha}}}-\frac{e^{\frac{x s}{\sqrt{\alpha}}}}{2 s \sqrt{\alpha}} \int\left[(g(x)+s f(x)) e^{\frac{-x s}{\sqrt{\alpha}}}\right] d x+\frac{e^{\frac{-x s}{\sqrt{\alpha}}}}{2 s \sqrt{\alpha}} \int\left[(g(x)+s f(x)) e^{\frac{x s}{\sqrt{\alpha}}}\right] d x
$$

Taking the inverse Laplace transform, we have the final solution as

$$
i(x, t)=k_{1} \delta\left(t+\frac{x}{\sqrt{\alpha}}\right)+k_{2} \delta\left(t-\frac{x}{\sqrt{\alpha}}\right)-L^{-1}\left[\frac{e^{\frac{x s}{\sqrt{\alpha}}}}{2 s \sqrt{\alpha}} \int\left[(g(x)+s f(x)) e^{\frac{-x s}{\sqrt{\alpha}}}\right] d x+L^{-1}\left[\frac{e^{\frac{-x s}{\sqrt{\alpha}}}}{2 s \sqrt{\alpha}} \int\left[(g(x)+s f(x)) e^{\frac{x s}{\sqrt{\alpha}}}\right] d x\right.\right.
$$

where $\delta(t)$ is the Dirac delta function [10]

\section{Computational Examples}

\subsection{Example1}

Let us consider the wave equation on transmission line with negligible leakage to ground on the line that is given by

$$
\frac{\partial^{2} i(x, t)}{\partial x^{2}}=\frac{\partial i(x, t)}{\partial t}
$$

with the initial conditions

$$
i(x, 0)=e^{x}, i_{t}(x, 0)=-e^{x},
$$

where $0<x<k$ and $\mathrm{k}$ is any given constant.

Taking the Laplace transform of the equation with respect to $t$ and substituting the initial conditions, we have

$$
s^{2} I(x, s)-s e^{x}+e^{x}=\frac{d^{2} I(x, s)}{d x^{2}}
$$

That is

$$
\frac{d^{2} I(x, s)}{d x^{2}}-s^{2} I(x, s)=\left(e^{x}-s e^{x}\right)
$$

Solving the associated homogeneous differential equation for the problem above, we have the complementary function as

$$
I_{c}(x, s)=k_{1} e^{x s}+k_{2} e^{-x s}
$$

Using the method of variation of parameters, we seek a particular solution of the form

$$
I_{p}(x, s)=U_{1}(x) I_{c_{1}}(x, s)+U_{2}(x) I_{c_{2}}(x, s)
$$

where $I_{c_{1}}(x, s)=e^{s x}, I_{c_{2}}(x, s)=e^{-s x}, U_{1}(x)=\int \frac{w_{1}}{w} d x$ and
$U_{2}(x)=\int \frac{w_{2}}{w} d x$

Values of $w, w_{1}$ and $w_{2}$ are obtained from the determinants below

$$
\begin{gathered}
w=\left|\begin{array}{cc}
e^{s x} & e^{-s x} \\
s e^{s x} & -s e^{-s x}
\end{array}\right| \\
w_{1}=\left|\begin{array}{cc}
0 & e^{-s x} \\
\left(e^{x}-s e^{x}\right) & -s e^{-s x}
\end{array}\right| \\
w_{2}=\left|\begin{array}{cc}
e^{s x} & 0 \\
s e^{s x} & \left(e^{x}-s e^{x}\right)
\end{array}\right|
\end{gathered}
$$

Therefore $\quad U_{1}(x)=\int \frac{\left(e^{x}-s e^{x}\right) e^{-s x}}{2 s} d x$ and $U_{2}(x)=$ $-\int \frac{\left(e^{x}-s e^{x}\right) e^{s x}}{2 s} d x$.

Substituting all these values in the equation

$$
I_{p}(x, s)=U_{1}(x) I_{c_{1}}(x, s)+U_{2}(x) I_{c_{2}}(x, s)
$$

we have the particular solution for this problem as

$$
I_{p}(x, s)=\frac{e^{x}}{2 s}+\frac{(s-1) e^{x}}{2 s(s+1)}
$$

The general solution of the problem is therefore

$$
I(x, s)=k_{1} e^{s x}+k_{2} e^{-s x}+\frac{e^{x}}{2 s}+\frac{(s-1) e^{x}}{2 s(s+1)}
$$

Simplifying further, we have

$$
I(x, s)=k_{1} e^{s x}+k_{2} e^{-s x}+\frac{e^{x}}{(s+1)}
$$

Taking the inverse Laplace transform, we have the final solution as

$$
i(x, t)=k_{1} \delta(t+x)+k_{2} \delta(t-x)+e^{(x-t)}
$$

where $\delta(t)$ is the Dirac delta function 


\subsection{Example 2}

Consider the wave equation on transmission line with very small leakage to ground on the line that is given by

$$
\frac{\partial^{2} i(x, t)}{\partial x^{2}}=\frac{\partial i(x, t)}{\partial t}
$$

with the initial conditions

$$
i(x, 0)=e^{x}, i_{t}(x, 0)=x, 0<x<k
$$

where $\mathrm{k}$ is any given constant.

Substituting the initial conditions of this problem into the general solution in (20) with $\alpha=1$, we have the general solution as

$$
\begin{gathered}
I(x, s)=k_{1} e^{x s}+k_{2} e^{-x s}-\frac{e^{x s}}{2 s} \int\left[\left(x+s e^{x}\right) e^{-x s}\right] d x+ \\
\frac{e^{-x s}}{2 s} \int\left[\left(x+s e^{x}\right) e^{x s}\right] d x
\end{gathered}
$$

where $k_{1} e^{x s}+k_{2} e^{-x s}$ is the complementary function and $-\frac{e^{x s}}{2 s} \int\left[\left(x+s e^{x}\right) e^{-x s}\right] d x+\frac{e^{-x s}}{2 s} \int\left[\left(x+s e^{x}\right) e^{x s}\right] d x$ is the particular solution.

Integrating the last two terms of the general solution and simplifying, we have

$$
I(x, s)=k_{1} e^{x s}+k_{2} e^{-x s}+\frac{x}{s^{2}}+\frac{e^{x}}{2(s-1)}+\frac{e^{x}}{2(s+1)}
$$

Taking the inverse Laplace transform, we have the final solution as

$$
I(x, s)=k_{1} e^{x s}+k_{2} e^{-x s}-\frac{\operatorname{Cos} x}{4}\left[\frac{1}{(s-1)}\right]+\frac{\operatorname{Cos} x}{4}\left[\frac{1}{(s+1)}\right]-\frac{\sin x}{4}\left[\frac{1}{(s-1)}\right]-\frac{\sin x}{4}\left[\frac{1}{(s+1)}\right]-\frac{\operatorname{Cos} x}{2}\left[\frac{1}{\left(s^{2}+1\right)}\right]+\frac{\operatorname{Sin} x}{2}\left[\frac{s}{\left(s^{2}+1\right)}\right]
$$

Taking the inverse Laplace transform, we have the final solution as

$$
i(x, t)=k_{1} \delta(t+x)+k_{2} \delta(t-x)-\frac{\operatorname{Cos} x e^{t}}{4}+\frac{\operatorname{Cos} x e^{-t}}{4}-\frac{\operatorname{Sin} x e^{t}}{4}-\frac{\operatorname{Sin} x e^{-t}}{4}-\frac{\operatorname{Cos} x \operatorname{Sin} t}{2}+\frac{\operatorname{Sin} x \operatorname{Cos} t}{2}
$$

where $\delta(t)$ is the Dirac delta function

\subsection{Example 4}

Consider the wave equation on transmission line with very small leakage to ground on the line that is given by

$$
\begin{aligned}
& \begin{array}{cc}
\frac{\partial^{2} i(x, t)}{\partial x^{2}}=4 \frac{\partial i(x, t)}{\partial t} & \begin{array}{c}
\text { general solution in }(20) \text { with } \alpha=4, \text { we have the general } \\
\text { solution as }
\end{array} \\
I(x, s)=k_{1} e^{\frac{x s}{2}}+k_{2} e^{\frac{-x s}{2}}-\frac{e^{\frac{x s}{2}}}{4 s} \int\left[\left(e^{-x}+s e^{x}\right) e^{\frac{-x s}{2}}\right] d x+\frac{e^{-\frac{x s}{2}}}{4 s} \int\left[\left(e^{-x}+s e^{x}\right) e^{\frac{x s}{2}}\right] d x=k_{1} e^{\frac{x s}{2}}+k_{2} e^{\frac{-x s}{2}}-\frac{e^{\frac{x s}{2}}}{4 s}\left\{\int e^{-x\left(1+\frac{s}{2}\right)} d x+\right.
\end{array} \\
& \begin{array}{cc}
\frac{\partial^{2} i(x, t)}{\partial x^{2}}=4 \frac{\partial i(x, t)}{\partial t} & \begin{array}{c}
\text { general solution in (20) with } \alpha=4, \text { we have the general } \\
\text { solution as }
\end{array} \\
I(x, s)=k_{1} e^{\frac{x s}{2}}+k_{2} e^{\frac{-x s}{2}}-\frac{e^{\frac{x s}{2}}}{4 s} \int\left[\left(e^{-x}+s e^{x}\right) e^{\frac{-x s}{2}}\right] d x+\frac{e^{-\frac{x s}{2}}}{4 s} \int\left[\left(e^{-x}+s e^{x}\right) e^{\frac{x s}{2}}\right] d x=k_{1} e^{\frac{x s}{2}}+k_{2} e^{\frac{-x s}{2}}-\frac{e^{\frac{x s}{2}}}{4 s}\left\{\int e^{-x\left(1+\frac{s}{2}\right)} d x+\right.
\end{array} \\
& \left.s \int e^{x\left(1-\frac{s}{2}\right)} d x\right\}+\frac{e^{\frac{-x s}{2}}}{4 s}\left\{\int e^{x\left(\frac{s}{2}-1\right)} d x+s \int e^{x\left(\frac{s}{2}+1\right)} d x\right\}
\end{aligned}
$$

where $k_{1} e^{\frac{x s}{2}}+k_{2} e^{\frac{-x s}{2}}$ is the complementary function and $-\frac{e^{\frac{x s}{2}}}{4 s}\left\{\int e^{-x\left(1+\frac{s}{2}\right)} d x+s \int e^{x\left(1-\frac{s}{2}\right)} d x\right\}+\frac{e^{\frac{-x s}{2}}}{4 s}\left\{\int e^{x\left(\frac{s}{2}-1\right)} d x+\right.$ $\left.s \int e^{x\left(\frac{s}{2}+1\right)} d x\right\}$ is the particular solution.

Integrating all the integrands in the general solution and simplifying, we have

$$
I(x, s)=k_{1} e^{\frac{x s}{2}}+k_{2} e^{\frac{-x s}{2}}+\frac{e^{-x}}{4}\left[\frac{1}{s}-\frac{1}{(s+2)}\right]+\frac{e^{x}}{2}\left[\frac{1}{(s-2)}\right]+\frac{e^{-x}}{4}\left[\frac{1}{(s-2)}-\frac{1}{s}\right]+\frac{e^{x}}{2}\left[\frac{1}{(s+2)}\right]
$$

Taking the inverse Laplace transform, we have the final solution as

$$
i(x, t)=k_{1} \delta(t+x)+k_{2} \delta(t-x)+x t+\frac{e^{(x+t)}}{2}+\frac{e^{(x-t)}}{2}
$$

where $\delta(t)$ is the Dirac delta function

Consider the wave equation on transmission line with where $\mathrm{k}$ is any given constant.

Substituting the initial conditions of this problem into the general solution in (20) with $\alpha=1$, we have the general olution as

$$
\begin{gathered}
I(x, s)=k_{1} e^{x s}+k_{2} e^{-x s}-\frac{e^{x s}}{2 s} \int\left[(s \operatorname{Sin} x) e^{-x s}\right] d x+ \\
\frac{e^{-x s}}{2 s} \int\left[(s \operatorname{Sin} x) e^{x s}\right] d x
\end{gathered}
$$

where $k_{1} e^{x s}+k_{2} e^{-x s}$ is the complementary function and $-\frac{e^{x s}}{2 s} \int\left[(\operatorname{Sin} x) e^{-x s}\right] d x+\frac{e^{-x s}}{2 s} \int\left[(\operatorname{Sin} x) e^{x s}\right] d x \quad$ is the

Integrating the last two terms of the general solution and with the initial conditions

$$
i(x, 0)=e^{x}, i_{t}(x, 0)=e^{-x}, 0<x<k
$$

Substituting the initial conditions of this problem into the

$$
\frac{\partial^{2} i(x, t)}{\partial x^{2}}=\frac{\partial i(x, t)}{\partial t}
$$

with the initial conditions

$$
i(x, 0)=\operatorname{Sin} x, i_{t}(x, 0)=0,0<x<k
$$

where $\mathrm{k}$ is any given constant.

Taking the inverse Laplace transform, we have the final solution as 


$$
\begin{gathered}
i(x, t)=k_{1} \delta\left(t+\frac{x}{2}\right)+k_{2} \delta\left(t-\frac{x}{2}\right)+\frac{e^{-x}}{4}\left[1-e^{-2 t}\right]+\frac{e^{x+2 t}}{2}+\frac{e^{-x}}{4}\left[e^{2 t}-1\right]+\frac{e^{x-2 t}}{2}=k_{1} \delta\left(t+\frac{x}{2}\right)+k_{2} \delta\left(t-\frac{x}{2}\right)- \\
\frac{e^{-(x+2 t)}}{4}+\frac{e^{x+2 t}}{2}+\frac{e^{-(x-2 t)}}{4}+\frac{e^{x-2 t}}{2}
\end{gathered}
$$

where $\delta(t)$ is the Dirac delta function

\section{Conclusion}

Analytical results of some transmission line equations where leakage to ground are negligible were obtained in this paper. The conductance and the inductance, which are responsible for leakages on the line, are set to zero in the model of the general wave equation for the transmission line. The resulted partial differential equations were transformed to ordinary differential equations by using the Laplace transform method and the method of variation of parameters was thereafter used to get the particular solution to the problem.

\section{References}

[1] Mehta, V.K. and Mehta, R. (2008). Principles of Power Systems, S. Chand and Company Limited, New Delhi.

[2] Wadhwa, C.L. (2009). Electrical Power Systems, New Age International Limited, New Delhi.

[3] Gupta, B. D. (2009). Mathematical Physics, Vikas Publishing House PVT Limited.

[4] Ajayi, E.O. (2009). Fourier and Hankel Transforms for Solving Boundary Value Problems, Deoban International Journal of Mathematical Sciences 11(3): 160 - 174.
[5] Ezekiel, F.D. and Ojo, S.O. (2008). An Integral Transform Method for Solving Boundary Value Problems, Deoma International of Sciences 13(2): $106-120$.

[6] Dass, H. K. and Verma, R. (2011). Mathematical Physics, S. Chand and Company Limited, New Delhi.

[7] Hayt, W.H. and Buck, J.A. (2006). Engineering Electromagnetics, McGraw-Hill Company Inc.

[8] Oke, M. O. (2012). Mathematical Model for the Determination of Voltage and Current on Lossy Power Transmission Line, IOSR Journal of Mathematics 1( 4): 16 18.

[9] Gupta, J.B. (2008). A Course in Power Systems, S.K. Kataria \& Sons, New Delhi.

[10] Stroud, K. A. and Dexter, J.B. (2003). Advanced Engineering Mathematics, Palgrave Macmillan Limited, New York.

[11] Bhattacharyya, B. (2009). Mathematical Physics, New Central Book Agency Limited, New Delhi.

[12] Zill, D.G. and Cullen, R. M. (2005). Differential Equations with Boundary-Value Problems, Brooks/Cole, Thomson Learning Inc., Canada.

[13] Riley, K. F., Hobson, M. P. and Bence, S.J. (2002). Mathematical Methods for Physics and Engineering, Cambridge University Press, New York. 